\title{
Caracterización molecular en aislados chilenos de Streptococcus agalactiae
}

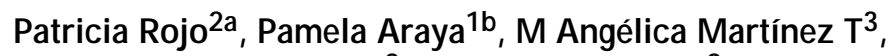 \\ Juan Carlos Hormazábal'2, Aurora Maldonado2a, \\ Jorge Fernández ${ }^{1 c}$. \\ Molecular characterization \\ of Chilean isolates \\ of streptococcus agalactiae
}

Background: Streptococcus agalactiae is the main causing organism of invasive infections such as sepsis and meningitis in the newborn. Aim: To perform a genotype characterization of Streptococcus agalactiae strains coming form invasive infections of newborns and colonized pregnant women. Material and methods: A group of 58 strains not related epidemiologically, isolated from colonized pregnant women and invasive infections in newborns, were studied. Pulsed field electrophoresis (PFGE) and polymerase chain reaction amplification of hylB and IS1548 genes, as possible virulence markers, were performed. Results: Among the studied strains, 37 genetic subtypes were observed. There were nine groups of identical PFGE patterns. Three corresponded to serotype Ia and six to serotype III. An erythromycin and clindamycin resistant clone was identified in three colonized women and a newborn with sepsis, which were not epidemiologically related. The hylB gene was equally present in cases of neonatal meningitis or colonized pregnant women. Conclusions: There was a great degree of polymorphism among the studied strains. The ample presence of hylB gene and the absence of the insertion element IS1548 in the hylB gene in invasive and colonizing strains, indicates that both groups of strains are potentially pathogenic (Rev Méd Chile 2008; 136: 606-12).

(Key words: Clindamycin; Drug resistance, bacterial; Erythromycin; Streptococcus agalactiae)

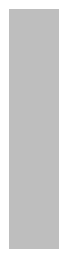

Recibido el 3 de enero, 2007. Aceptado el 28 de enero, 2008.

Trabajo financiado con fondos del Instituto de Salud Pública de Chile.

${ }^{1}$ Genética Molecular y ${ }^{2}$ Sección Bacteriología Clínica, Instituto de Salud Pública de Chile.

3Instituto de Ciencias Biomédicas (ICBM) Universidad de Chile. Santiago de Chile.

aTecnólogo Médico

bioquímico

CPhD y Licenciado en Biología

Correspondencia a: Dr. Jorge Fernández Ordenes. Genética Molecular, Departamento Laboratorios Biomédicos Nacional de Referencia, Instituto de Salud Pública de Chile. Marathon 1000, Ñunoa, Santiago de Chile. Fax: 3507573.

E mail: Jfernand@ispch.cl 
$E^{1}$ Streptococcus agalactiae (SGB) es una cocácea grampositiva, beta-hemolítica que presenta un polisacárido común que lo clasifica como perteneciente al grupo B de Lancefield ${ }^{1}$. Actualmente es el agente etiológico más frecuente responsable de sepsis, meningitis y neumonía neonatal ${ }^{2,3}$. El SGB forma parte de la flora normal del tracto gastrointestinal y puede colonizar tracto genitourinario, lo que es un hecho importante en las gestantes, por la posibilidad de transmisión del $\mathrm{S}$ agalactiae al recién nacido ${ }^{1-4}$. Streptococcus agalactiae se ha clasificado en nueve serotipos (Ia, Ib y II-VIII), basado en los antígenos de polisacáridos capsulares y antígenos proteicos ${ }^{5,6}$. Este microorganismo es susceptible a penicilina y a otros antibióticos ß-lactámicos. En el último tiempo han emergido cepas resistentes a eritromicina y clindamicina, antibióticos de uso alternativo en la quimioprofilaxis intraparto de pacientes alérgicas a penicilina ${ }^{7-9}$.

Se han desarrollado diferentes métodos de genotipificación de cepas SGB para determinar clones virulentos, como el análisis de los patrones de amplificación al azar (RAPD) ${ }^{10}$, electroforesis enzima multilocus (MLEE) 11,12 , electroforesis en campo pulsado (PFGE) ${ }^{13,14}$, reacción en cadena de la polimerasa (RCP) de genes virulentos ${ }^{15,16}$.

Uno de los genes potencialmente asociado con la virulencia de S agalactiae es el gen hylB que codifica para la enzima hialuronato liasa, que degrada el ácido hialurónico facilitando la diseminación bacteriana. Se ha descrito que el gen de esta enzima es inactivado por la introducción del elemento de inserción de 1317 nucleótido (IS1548) $^{17,18}$, disminuyendo la virulencia en estas cepas.

El objetivo de este estudio fue caracterizar genotípicamente cepas chilenas de Streptococcus agalactiae, provenientes de infecciones invasoras en recién nacidos y mujeres embarazadas colonizadas, mediante electroforesis en campo pulsado y reacción en cadena de la polimerasa.

\section{MateRIAL y MÉTOdo}

Cepas bacterianas. Se estudió un total de 58 cepas de Sagalactiae aisladas entre los años 1998 y 2005, 13 comespondieron a cepas provenientes de meningitis, 13 sepsis neonatal, 1 síndrome febril, 4 sin antecedentes clínicos en recién nacidos. Treinta y tres cepas correspondieron a una colección del Laboratorio de Referencia de Cocáceas Gram positivas del Instituto de Salud Pública de Chile, obtenidas desde el Hospital de Curicó, Hospital el Pino, Hospital San Borja Arriarán, Hospital Félix Bulnes, Hospital de Arica, Hospital de Ovalle, Hospital de Coquimbo, Hospital de Chillán, Hospital de Punta Arenas, Hospital de Buin, Hospital de Concepción, Hospital de Talagante, Hospital Exequiel González Cortés, Hospital Luis Calvo Mackenna. Las 25 cepas restantes correspondieron a cepas colonizantes vaginales-perianales provenientes de mujeres embarazadas pertenecientes a una colección del Instituto de Ciencias Biomédicas (ICBM) de la Universidad de Chile.

Serotipificación. A cada aislamiento se le realizó confirmación mediante pruebas bioquímicas: test de CAMP e hidrólisis de hipurato de sodio positivo y ausencia de crecimiento en agar bilis esculina. Las cepas fueron clasificadas en serotipos Ia, Ib, II, III, IV y V, mediante aglutinación con antisueros comerciales (Denka, Seiken, Tokio, Japón) de acuerdo a las instrucciones del fabricante.

Susceptibilidad. A todas las cepas se les determinó la concentración mínima inhibitoria a penicilina, eritromicina, clindamicina y vancomicina, por método de microdilución en caldo según estándar CLSI $^{19}$.

Análisis cromosomal por electroforesis en campo pulsado (PFGE). La extracción del ADN cromosomal se realizó según protocolo modificado de Benson $^{20}$. Los aislados fueron crecidos en placas de agar sangre por $24 \mathrm{~h}$ a $37^{\circ} \mathrm{C}$, luego las células bacterianas se resuspendieron en tampón TE (10 mM Tris, 1 mM EDTA, pH 8,0) y la suspensión fue incubada con $10 \mu \mathrm{l}$ de lisozima $(10 \mathrm{mg} / \mathrm{ml})$ por 15 min a $37^{\circ} \mathrm{C}$. Posteriormente, la suspensión bacteriana fue mezclada con $10 \mu \mathrm{l}$ mutanolisina (1 mg/ $\mathrm{ml}), 5 \mu \mathrm{l}$ de proteinasa $\mathrm{K}(20 \mathrm{mg} / \mathrm{ml})$ y agarosa de bajo punto de fusión al $2 \%$. Los moldes de agarosa fueron incubados con tampón de lisis $(0,5 \mathrm{mM}$ EDTA, 1\% lauryl sarcosina pH 8,0) y $30 \mu \mathrm{lde}$ proteinasa $\mathrm{K}(20 \mathrm{mg} / \mathrm{ml})$ durante $2 \mathrm{~h}$ a $55^{\circ} \mathrm{C}$ con agitación. Luego, los moldes fueron lavados tres veces con agua destilada estéril y tampón TE por 15 min a 55ํ․ Una porción del molde de agarosa 
fue digerido con $10 \mathrm{U}$ de enzima SmaI durante 4 h. La electroforesis de campo pulsado fue desarrollada en un equipo CHEF DR III (BioRad) en agarosa al 1\% (calidad PFGE) y con solución TBE (4,5 mM Tris-HCL, 4,5 mM ácido bórico, 0,125 mM EDTA $\{\mathrm{pH} 7,6\}$. Las condiciones de electroforesis fueron: pulsos iniciales de $8 \mathrm{~s}$; pulsos finales de 30 s; voltaje $6 \mathrm{~V} / \mathrm{cm}$, ángulo $120^{\circ}$, duración $23 \mathrm{~h}$ a $14^{\circ}{ }^{20}$. Los patrones de digestión PFGE fueron detectados en sistema documentación de geles Gel DOC 2000 de BioRad, y el análisis filogenético se realizó en el programa bioinformático Molecular Analyst de BioRad.

Detección del gen hylB y elemento de inserción 1548. El gen que codifica para la proteína hialuronato liasa hylB se amplificó mediante la reacción en cadena de la polimerasa ${ }^{15}$. La reacción de RCP fue efectuada en un volumen final de $50 \mathrm{ml}$ consistente en $1,5 \mathrm{mM}$ de $\mathrm{MgCl}_{2}, 20 \mathrm{mM}$ Tris- $\mathrm{HCl}$ pH 8,4, $50 \mathrm{mM} \mathrm{KCl}, 10 \%$ glicerol, 2 pmoles de los partidores hylBfw (5'AGCCACTCATAGCACAATGAAACAAAG3') y hylBrev (5'CTAGTTAGATAGCTAATTGGTCTGTTT $3^{\prime}$ ), $200 \mathrm{mM}$ dNTP, $1 \mathrm{U}$ de Taq polimerasa y $5 \mathrm{ml}$ de lisado celular como templado. El proceso de amplificación del ADN consistió en una etapa de denaturación inicial de $95^{\circ} \mathrm{C}$ por 5 min, seguida por 30 ciclos de amplificación que incluyeron 1 min de denaturación a $94^{\circ} \mathrm{C}, 1$ min de hibridación a $60^{\circ} \mathrm{C}$ y 1 min de extensión a $72^{\circ} \mathrm{C}$, para terminar con etapa de extensión final de $7 \mathrm{~min}$ a $72^{\circ} \mathrm{C}$. El elemento de inserción IS 1548 se amplificó con los partidores IS1548 fw (5'CCAAACGCATGAAGAAACTTTTGTTTTCAG3') y IS1548 rev (5'CTTCATCCTTTTGTGCCCGGACATCA3'), con los mismos ciclos descritos anteriormente ${ }^{15}$. Los productos de PCR fueron analizados por electroforesis en geles de agarosa al $1 \%$ y visualizados en transiluminador de luz UV.

\section{Resultados}

La diversidad genética de 58 cepas de Streptococcus agalactiae aisladas de mujeres embarazadas colonizadas y de recién nacidos con sepsis y meningitis, se estudió mediante electroforesis en campo pulsado utilizando la enzima SmaI. Se observaron 37 patrones de PFGE diferentes, los cuales tenían un mínimo de 7 bandas y máximo de 10 bandas. En la Figura 1 se observan los patrones de PFGE obtenidos y la relación filogenética que existe entre ellos.
Figura 1: Relación genética de las 58 cepas de Streptococcus agalactiae aisladas en mujeres embarazadas colonizadas y de recién nacidos. Se observan 9 grupos clonales denominados desde A-I.

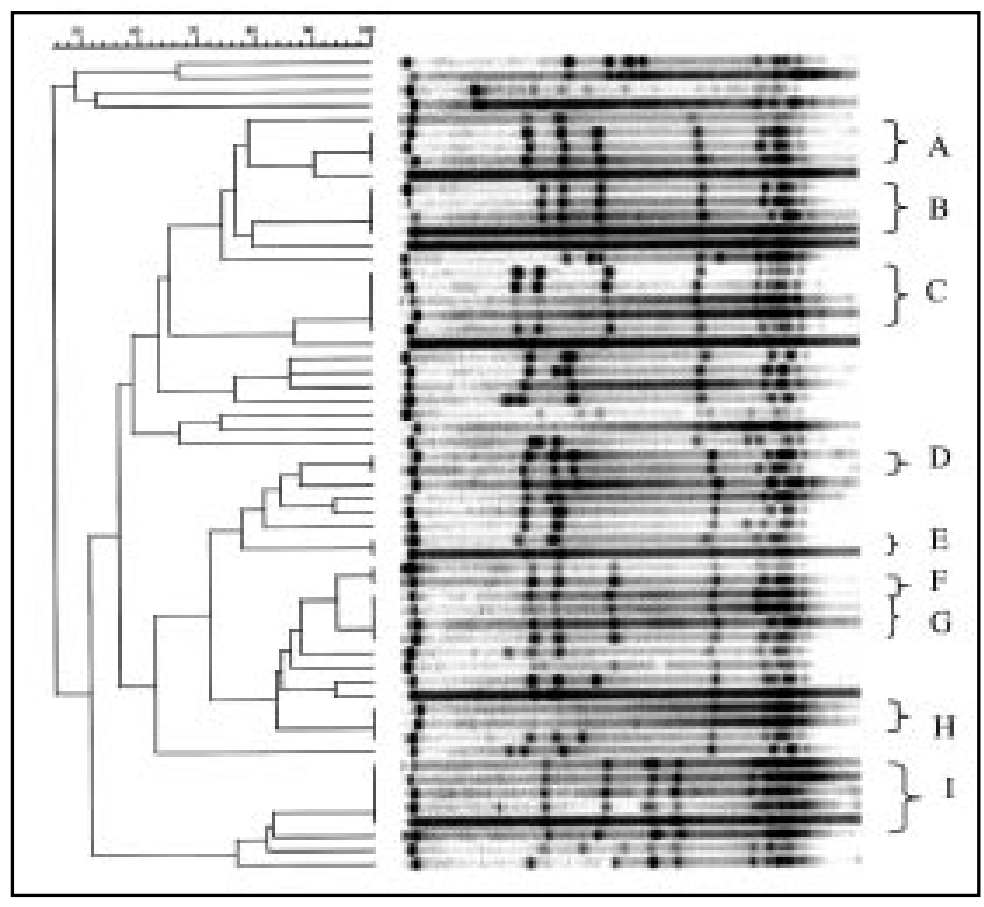


Los patrones de PFGE dentro de cada serotipo difieren considerablemente, en el serotipo III se observó un alto grado de heterogeneidad con 21 subtipos genéticos en las 33 cepas analizadas, el serotipo Ia con 9 subtipos en 18 cepas y el serotipo II con 5 subtipos en las 5 cepas estudiadas. En los serotipos Ib y V se encontró un subtipo, respectivamente (Tabla 1). En 27 cepas obtenidas de mujeres colonizadas se encontraron 21 subtipos, 12 subtipos fueron identificados desde 16 cepas de S Agalactiae aisladas desde muestras de sangre, 13 subtipos se encontraron a partir de 14 cepas aisladas de LCR (Tabla 2). De 37 subtipos encontrados, dos subtipos se detectaron en madres colonizadas, sangre y LCR.

El análisis del dendrograma mostró 9 agrupamientos idénticos denominándolos arbitrariamente desde A-I (Figura 1). En los agrupamientos A, B, e I se encontraron sólo aislados del serotipo Ia provenientes de mujeres embarazadas colonizadas y de recién nacidos con sepsis y meningitis. En los agrupamientos C, D, E, F, G y H se observaron sólo cepas pertenecientes al serotipo III, provenientes de recién nacidos con meningitis, sepsis y mujeres colonizadas. En el agrupamiento $G$ se identificó el mismo patrón PFGE en cepas obtenidas de una madre colonizada y sus 2 hijos gemelos con sepsis. El agrupamiento C consistió en cepas que provenían de mujeres colonizadas resistentes a eritromicina y clindamicina, este grupo está estrechamente relacionado a una cepa del serotipo III proveniente de un recién nacido con sepsis, con el mismo patrón de resistencia.

La detección del gen hylB que codifica para la hialurinato liasa se realizó mediante la amplificación de un fragmento de 2,9 kb por RCP. En todas las cepas provenientes de mujeres colonizadas y recién nacidos con alguna patología se detectó el gen hylB. En ninguna de las cepas estudiadas se identificó el elemento de inserción IS1548, que provoca la inactivación de la enzima hialurinato liasa.

\section{DISCUSIÓN}

La tipificación molecular de S agalactiae tiene ventajas sobre las serotipificación tradicional, ya que permite identificar fuentes de infección en la enfermedad neonatal temprana y tardía, también

Tabla 1. D iversidad genética en las 58 cepas de Streptococcus agalactiae agrupadas de acuerdo a los serotipos

\begin{tabular}{|ccc|}
\hline Serotipos & no de cepas & no de patrones de PFGE dentro de cada serotipo \\
\hline Ia & 18 & 9 \\
Ib & 1 & 1 \\
II & 5 & 5 \\
III & 33 & 21 \\
IV & 0 & 0 \\
V & 1 & 1 \\
\hline
\end{tabular}

Tabla 2. D iversidad genética en las 58 cepas de Streptococcus agalactiae agrupadas de acuerdo al origen

\begin{tabular}{|lcc|}
\hline Origen & № de cepas & № de patrones de PFGE \\
\hline Colonizadas & 27 & 21 \\
Sangre & 14 & 13 \\
LCR & 16 & 12 \\
Contenido gástrico & 1 & 1 \\
\hline
\end{tabular}


ha sido una importante herramienta en la investigación de brotes nosocomiales ${ }^{13,20,21}$. Los patrones de PFGE obtenidos mediante la digestión con SmaI en cepas S agalactiae, son fácilmente interpretados a diferencias de otros métodos tradicionales de electroforesis.

En este estudio, nosotros tipificamos cepas de $\mathrm{S}$ agalactiae aisladas desde madres embarazadas colonizadas y niños recién nacidos con sepsis, mediante análisis de PFGE. Los resultados demostraron una amplia diversidad genética en cepas aisladas desde madres embarazadas colonizadas y niños recién nacidos, similar a los encontrados en otros países ${ }^{22,23}$. Los serotipos Ia y III predominantes en Chile mostraron un alto grado de heterogeneidad genética ${ }^{24}$.

La transmisión y la adquisición de Sagalactiae en un recién nacido pueden ser consideradas algo normal entre madre e hijo como se observó en este trabajo donde una madre colonizada tenía el mismo patrón genético que sus hijos, sin embargo, no se puede descartar la posibilidad de que otras rutas de transmisión puedan existir, debido a que en muchos niños recién nacidos con sepsis el patrón de PFGE de S agalactiae aislado no es el mismo patrón presente en la madre colonizada ${ }^{25}$.

Los resultados de susceptibilidad antimicrobiana son similares a los encontrados en otros estudios con la aparición de la resistencia a eritromicina y clindamicina, antibióticos alternativos en la quimioprofilaxis de pacientes alérgicas a penicilina $^{26-28}$. Los patrones de PFGE de 3 cepas serotipo III resistentes a eritromicina y clindamicina fueron idénticos y estrechamente relacionados a otra cepa de $\mathrm{RN}$ del serotipo III resistente a los mismos antibióticos. Este hallazgo, podría indicar que un clon resistente está circulando en nuestro país. Diversos estudios de genotipificación basados en métodos de multilocus enzimático y electroforesis de fragmento polimórficos (RLFP) han sugerido que un subgrupo de S agalactiae serotipo III es particularmente invasivo ${ }^{29}$. Posteriormente, Takahashi et $\mathrm{al}^{31}$ y Bohnsack et $\mathrm{al}^{32}$ identificaron en Japón y Estados Unidos de Norteamérica un patrón específico de PFGE, asociado con altos niveles de producción de ácido siálico, presente en niños recién nacidos con meningitis. Al comparar nuestros patrones de PFGE de cepas de $\mathrm{S}$ agalactiae chilenas serotipo III, aislados en neonatos con meningitis, no se observó ninguna similitud con el patrón descrito, lo que demuestra que en nuestro país, este patrón no es predominante en las meningitis provocadas por S agalactiae en niños recién nacidos. Sólo se observa un patrón similar de PFGE, en una cepa de $\mathrm{S}$ agalactiae serotipo III aislada de una madre embarazada colonizada.

Se ha descrito que el gen hyl B, es un importante marcador de virulencia, se ha identificado preferentemente en cepas aisladas de cuadros invasivos, sin embargo cuando S agalactiae tiene inactivado este gen, por el elemento de inserción IS1548, las cepas no son patogénicas ${ }^{15,17}$. El análisis de nuestros aislados demostró que en todos los casos de meningitis y sepsis el gen hyl B estaba presente. Sin embargo, este gen también fue encontrado activo en madres embarazadas colonizadas. La presencia de este factor de virulencia en madres embarazadas colonizadas y neonatos con meningitis y sepsis podría indicar que otros factores son los que determinan el desarrollo de la enfermedad. Diversos estudios han demostrado que factores como el intervalo entre la ruptura de la membrana y el parto, parto prematuro, bajo peso del recién nacido, densa colonización vagino-rectal y fiebre intraparto son factores de riesgo importante para contraer la enfermedad $^{32,33}$. Otro aspecto importante es la susceptibilidad del recién nacido a la infección por SGB debido a una baja en los niveles de anticuerpos maternos contra la cápsula del polisacárido o las proteínas de la superficie.

El serotipo V ha emergido en Estados Unidos de Norteamérica, Europa y Asia desde el año 1990, provocando infecciones invasivas en neonatos y adultos ${ }^{34}$. En nuestro país se observó la aparición del serotipo $\mathrm{V}$ en una cepa aislada en recién nacido con infección invasiva. El patrón de PFGE de esta cepa serotipo V, demostró que es similar a los patrones de PFGE descritos en otros países $^{35,36}$. La genotipificación por electroforesis de campo pulsado permitió conocer y comparar los distintos subtipos de S agalactiae que circulan en nuestro país. Este estudio confirma la importancia de la creación y mantenimiento de librerías genéticas asociadas a enfermedad infecciosas relevantes en salud pública. 


\section{REFERENCIAS}

1. CReSPo M, Vélez J. Importancia clínica de Streptococcus agalactiae como causante de infección. Colombia Médica 1997; 27: 53-8.

2. Valdés R, Pastene $S$, Morales A, Gutiérrez B, Canales A, Martínez P et al. Prevalencia de colonización por Streptococcus agalactiae (grupo B) durante el embarazo pesquisado en medio de cultivo selectivo. Rev Chil Obstet Ginecol 2004; 69: 132-5.

3. Foxman B, Gilespie B, Manning S, Howard L, TaLman P, Zhang L, MarRs C. Incidence and duration of group B Streptococcus by serotype among male and female college students living in a single dormitory. Am J Epidemiol 2006; 163: 544-51.

4. CDC. Prevention of perinatal group B streptococcal disease. MMWR 2002/51 (RR11); 1-22.

5. Martínez MA, Ovalue A, Durán C, Reid I, Urriola G, Garay B, Cifuentes M. Serotipos y suceptibilidad antimicrobiana de Streptococcus agalatiae. Rev Méd Chile 2004; 132: 549-55.

6. Moyo S, Maeland J, Bergh K. Typing of human isolates of Streptococcus agalactiae (group B streptococcus, GBS) strains from Zimbabwe. J Med Microbiol 2002; 51: 595-600.

7. De Mouy D, Cavalo J-D, Leclerce R, Fabre R, and THE AFORCOPI-BIO NETwORK. Antibiotic susceptibility and mechanisms of erythromycin resistance in clinical isolates of Streptococcus agalactiae: French Multiautor agents chemother. 2001; 45: 2400-2.

8. von Both U, Ruess M, Muelier U, Fluegge K, Sander A, BERNER RA. A serotype $V$ clone is predominant among erythromycin-resistant Streptococcus agalactiae isolates in a southwestern region of Germany. J Clin Microbiol 2003; 41: 2166-9.

9. Wehbeh W, Rojas-Diaz R, L X, Mariano N, Grenner L, Segal-Maurer S et al. Fluoroquinolone-Resistant Streptococcus agalactiae: Epidemiology and mechanism of resistance. Antimicrob Agents Chemother 2006; 49: 2495-7.

10. Martínez G, Harel J, Higgins R, Lacouture S, Daignault D, GotTschalk M. Characterization of Streptococcus agalactiae isolated of bovine and human origin by randomly amplified polymorphic DNA analysis. J Clin Microbiol 2000; 38: 71-8.

11. Quentin R, Huet H, Wang F, Gesun P, Goudeau A, SELANDER R. Characterization of Streptococcus aga- lactiae strains by multilocus enzyme genotype and serotype: Identification of multiple virulent clone families that cause invasive neonatal disease. J Clin Microbiol 1995; 33: 2576-81.

12. Ramaswamy S, Ferrieri P, Flores A, Paoletti L. Molecular Characterization of nontypeable group B Streptococcus. J Clin Microbiol 2006; 44: 2398403.

13. Benson K, Luchansky J, Eunot J, Degnan A, WiLenBERG H, Thornbery J, Kay H. Pulsed-Field fingerprinting of vaginal group B streptococcus in pregnancy. Am J Obstet Gynecol 2002; 100: 545-51.

14. Oliveira IC, De Mattos MC, Areal MF, FerreiraCarvahlo BT, Figuiredo AM, Benchetrit LC. PulsedFiled gel electrophoresis of human group B streptococci isolated in Brazil. J Chemother 2005; 17: 258-63.

15. Rolad K, Marios C, Siquier V, Catire B, Quentin R. Genetic features of Streptococcus agalatiae strains causing severe neonatal infections, as revealed by pulsed-field gel electroforesis and hylB gene analysis. J Clin Microbiol 1999; 37: 1892-8.

16. Ramaswamy S, Ferrieri P, Madoff L, Flores A, Kumar $\mathrm{N}$, Tettelin $\mathrm{H}$, Paoletti L Identification of novel cps lócus polymprphisms in nontypable group B Streptococcus. Journal Medical Microbiology 2006; 55: 775-83.

17. Dmitriev A, Hu Y, Shen A, Suvorov A, Yang Y. Chromosomal analysis of group B streptococcal clinical strains; bac gene-positive strains are genetically homogenous. FEMS Microbiology Letters 2001; 208: 93-8.

18. Granlund M, Öberg L, Seumn M, Norgren M. Identification of a novel insertion element, IS 1548, in group B streptococci, predominantly in strains causing endocarditis. J Infect Dis 1998; 177: 967-76.

19. National Committee for Clinical Laboratory Standards. Performance standards for antimicrobial susceptibility testing: Twelfth informational Supplement. M2 - A9 Vol. 23 № 1, 2006.

20. Benson J, FerRIERI P. Rapid pulsed-field gel electrophoresis method for group B Streptococcus isolates. J Clin Microbiol 2001; 39: 3006-8.

21. Ekelund K, Slotved H, Nielsen H, Kaltoft M, KoradSEN H. Emergence of invasive serotype VIII group B Streptococcal infections in Denmark. J Clin Microbiol 2003; 41: 4442-4.

22. Skjaervold N, Bergh K, Bevanger L Distribution of PFGE of invasive group $B$ streptococcus in 
relation to serotypes. Indian J Med Res 2004; 119: 201-4.

23. Ekuland K, Sltved H, Nielsen H, Kaltoft M, Koradsen H. Emergence of invasive serotype VIII group Streptococcus infections in Denmark. J Clin Microbiol 2003; 41: 4442-4.

24. Lämmer C, Schawarz S, Wibawan IWT, Ott E, Bop B, MarTínEZ-TAgle A. Comparison of streptococcus of serological group B isolates from healthy carriers and active disease in Chile. J Med Microbiol 1995; 42: 161-4.

25. Fasola E, Lvddahl C, FerRIERI P. Molecular analysis of multiple isolates of the major serotypes of group B streptococci. J Clin Microbiol 1993; 31: 2616-20.

26. Roberts M, Sutclife J, Courvalin P, Jensen LB, Rood $J$, Seppala $H$. Nomenclature for macrolide and macrolide-lincosamide-streptogramin B resistance determinants. Antimicrob Agents Chemother 1999; 43: 2823-30.

27. Pearlman MD, Pierson CL, Faix RG. Frequent resistance in clinical group B streptococci isolates to clindamycin and erytromycin. Obstet Gynecol 1998; 92: 258-61.

28. Ko W, Yan J, Lee N, Wu H, Wu J. Polyclonal spread of eritromycin-resistant Streptococcus agalactiae in southem Taiwan. Microb Drug Resist 2004; 10: 306-12.

29. Ip M, Cheuk E, Tsui M, Kong F, Leung T, Gilbert G. Identification of a streptococcus agalactiae serotype III subtype 4 clone in association with adult invasive disease in Hong Kong. J Clin Microbiol 2006; 44: 4252-4.
30. Bidet P, Brahimi N, Chalas C, Aujard Y, Bingen E. Molecular characterization of serotype III group B Streptococcus isolates causing neonatal meningitis. J Infect Dis 2003; 188: 1132-7.

31. Takahashi S, Detrick S, Whiting AA, BlaschkeBonkowksy AJ, Aoyagi Y, Adderson EE, Bohnsack JF. Correlation of phylogenetic lineages of group B Streptococci, identified by analysis of restriction-digestion patterns of genomic DNA, with infB alleles and mobile genetic elements. J Infect Dis 2002; 1; 186: 1034-8.

32. Bohnsack JF, Whitting AA, Martínez G, Jones $N$, Adderson EE, Detrick S ET aL. Serotype III Streptococcus agalactiae from bovine milk and human neonatal infections. Emerg Infect Dis 2004; 10: 1412-9.

33. Lindahl G, Stalhammar-Carlemaln M, Areschoug T. Surface proteins of Streptococcus agalactiae and related proteins in other bacterial pathogens. Clin Microbiolo Reviews 2005; 18: 102-7.

34. EшOTT J, FARMER K, FACKIAM R. Sudden increase in isolation of group B streptococci, serotype $\mathrm{V}$, is not due to emergence of a new pulsed-field gel electrophoresis type. J Clin Microbiol 1998; 36: 2115-6.

35. Thomas-Bories I, Fitoussi F, Mariani-KuRKdjan P, RayMOND J, BRAHIMI N, BdDEt P ET AL. Clonal relationship between US and French serotype V group B Streptococcus isolates. J Clin Microbiol 2001; 39: 4526-8.

36. Amudson N, Flores A, Hiwer S, Baker C, Ferrieri P. DNA macrorestriction analysis of nontypeable group B streptococcal isolates: clonal evolution of nontypeable and type $\mathrm{V}$ isolates. J Clin Microbiol 2005; 43: 572-57. 